\title{
Pacific
}

Journal of

Mathematics

\section{BOUNDED AND UNBOUNDED CAPILLARY SURFACES IN A} CUSP DOMAIN

YASUNORI AOKI AND DAVID SIEGEL 


\title{
BOUNDED AND UNBOUNDED CAPILLARY SURFACES IN A CUSP DOMAIN
}

\author{
YASUNORI AOKI AND DAVID SIEGEL
}

\begin{abstract}
We study asymptotic behavior of the height of a static liquid surface in a cusp domain as modelled by the Laplace-Young capillary surface equation. We introduce a new form of an asymptotic expansion in terms of the functions defining the boundary curves forming a cusp. We are able to address the asymptotic behavior of the capillary surface in cusp domains not previously considered, such as an exponential cusp. In addition, we have shown that the capillary surface in a cusp domain is bounded if the contact angles of the boundary walls forming a cusp are supplementary angles, which implies the continuity of the capillary surface at the cusp.
\end{abstract}

\section{Introduction}

Background. In everyday life, it is often safe to assume that the surface of water at rest is almost flat; however, careful observation shows that the surface of water in a container can exhibit complicated geometry near the interface where the water meets the container. One of the most extreme examples is when the container has a sharp (cusped) boundary. As seen in the photo, the static liquid surface (capillary surface) rises very steeply near a cusp - formed in the case illustrated here by the tangency between a circular cylinder and a straight wall. This behavior can be understood through a singular solution of the Laplace-Young capillary surface equation.

As noted in [Finn 1986], the study of a singular capillary surface can be traced back to Brook Taylor in 1712. Later contributions to the study of singular capillary surfaces by Concus and Finn [1969] and Miersemann [1993] spurred considerable interest in the field; see, for example [King et al. 1999; Scholz 2001; 2004; Norbury et al. 2005; Aoki 2007]. In particular, Scholz's work on capillary surfaces in a

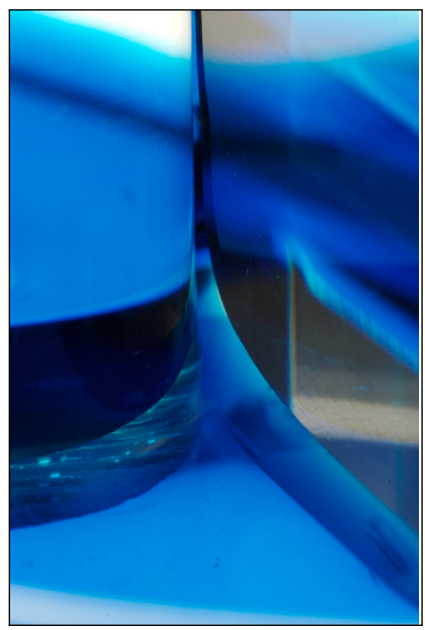

MSC2010: 35A20, 35C20, 35J60, 76B45.

Keywords: singularity, asymptotic analysis, nonlinear elliptic PDE. 
domain containing a cusp where the boundaries can be approximated by power series (including fractional powers) led him to conclude that "[the capillary surface] rises with the same order [as] the order of contact of the two arcs, which form the cusp" [Scholz 2004]. Since this is a is very intuitive statement, our curiosity led us to ask whether this statement holds for cases that Scholz did not consider in his paper [2004].

In this paper we extend Scholz's results in two directions. We first consider cusp domains not limited to the power-law cusp. Instead of approximating the boundary by power series, we directly use the distance between two arcs forming a cusp in the asymptotic expansion. Although one may argue that most of the shapes used in real life applications can be approximated by power series, our main focus was to justify the above statement in a more direct and intuitive manner, by avoiding the extra approximation step. The second direction of extension is to include cases in which the contact angles of the boundary walls forming a cusp are supplementary angles. Although all the known results suggest that a capillary surface in a domain with a cusp is unbounded, we have shown that a capillary surface can be bounded, and hence continuous, if the contact angles are supplementary angles.

Statement of the problems. Here we state the problems we are going to consider in this paper. We first define a cusp domain. Without loss of generality, and for simplicity of writing, we consider the following domain (see Figure 1):

$$
\Omega=\left\{(x, y): x>0, f_{2}(x)<y<f_{1}(x)\right\},
$$

where

$$
\begin{aligned}
f_{1}(x), f_{2}(x) \in C^{3}(0, \infty), & f_{1}(x)>f_{2}(x) \text { for } x>0, \\
\lim _{x \rightarrow 0^{+}} f_{1}(x)=\lim _{x \rightarrow 0^{+}} f_{2}(x)=0, & \lim _{x \rightarrow 0^{+}} f_{1}^{\prime}(x)=\lim _{x \rightarrow 0^{+}} f_{2}^{\prime}(x)=0 .
\end{aligned}
$$

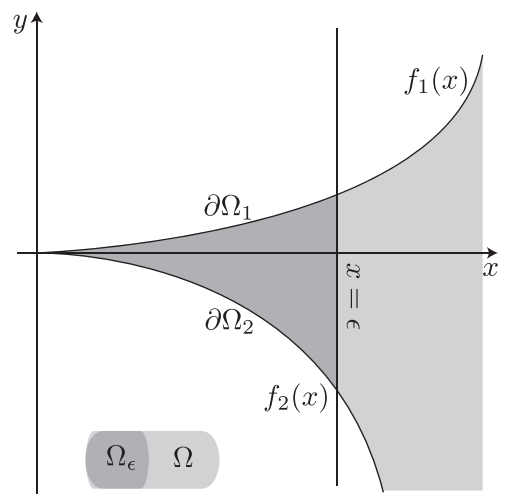

Figure 1. The cusped domain $\Omega$ and its boundary. 
Also we denote the boundaries as follows:

$$
\partial \Omega_{1}=\left\{(x, y): x>0, y=f_{1}(x)\right\}, \quad \partial \Omega_{2}=\left\{(x, y): x>0, y=f_{2}(x)\right\} .
$$

Although we base our dicussion on this infinite domain, all of the results presented in this paper only depend locally on a domain sufficiently close to the cusp, so the results hold for any domain that coincides with $\Omega$ in a neighborhood of the origin.

We now state the partial differential equation that interests us, the LaplaceYoung capillary surface equation. Let $u(x, y)$ be the height of a capillary surface in domain $\Omega$. It satisfies the following boundary value problem (see [Finn 1986] for a derivation):

$$
\begin{array}{ll}
\nabla \cdot T u=\kappa u & \text { in } \Omega, \\
\vec{v}_{1} \cdot T u=\cos \gamma_{1} & \text { on } \partial \Omega_{1}, \\
\vec{v}_{2} \cdot T u=\cos \gamma_{2} & \text { on } \partial \Omega_{2},
\end{array}
$$

where

$$
T u=\frac{\nabla u}{\sqrt{1+|\nabla u|^{2}}},
$$

$\kappa$ is the capillarity constant, $\vec{v}_{1}$ and $\vec{v}_{2}$ are exterior unit normal vectors on the boundaries $\partial \Omega_{1}$ and $\partial \Omega_{2}$, and $\gamma_{1}, \gamma_{2}$ are the contact angles. The capillarity constant $\kappa$ can be normalized by rescaling $x, y$, and $u$. In the sequel we let $\kappa=1$.

Here we introduce the big theta notation to replace the statement "is of the same order as", to make this expression more precise. If $f(x)=\Theta(g(x))$, there exist constants $k_{1}, k_{2}>0$ and $x_{0}>0$ such that

$$
k_{1}|g(x)|<|f(x)|<k_{2}|g(x)| \text { for all } x<x_{0} .
$$

We note that $\Theta$ is a more strict order relation than that of $O$, i.e., if $f(x)=\Theta(g(x))$ then $f(x)=O(g(x))$; however the converse is not true.

We can now write our core research questions as follows: - Suppose $\gamma_{1}+\gamma_{2} \neq \pi$. Does $u(x, y)=\Theta\left(\frac{1}{f_{1}(x)-f_{2}(x)}\right)$ hold for any $f_{1}(x)$
and $f_{2}(x)$ satisfying (1-2)?

- How does $u(x, y)$ behave asymptotically as $x \rightarrow 0^{+}$when $\gamma_{1}+\gamma_{2}=\pi$ ?

Structure of the paper. As the title of this paper suggests, there are two main parts: unbounded and bounded cases.

In Section 2 we consider unbounded capillary surfaces in cusp domains. We first prove in Section 2A that capillary surfaces are unbounded if $\gamma_{1}+\gamma_{2} \neq \pi$. Then in Section $2 \mathrm{~B}$ the formal asymptotic expansion is presented. Using the formal asymptotic expansion, in Section $2 \mathrm{C}$ we prove the asymptotic behavior of the 
solution. In Section 2D we give examples of power-law and non-power-law cusps with the intention of comparing our findings with the results in [Scholz 2004].

In Section 3 we consider bounded capillary surfaces in cusp domains. We first prove in Section 3A that capillary surfaces are bounded if $\gamma_{1}+\gamma_{2}=\pi$ and the curvature of the boundaries is finite. In Section $3 \mathrm{~B}$ we show that if a capillary surface is bounded at the cusp, then it is continuous at the cusp. Section 4 contains concluding remarks summarizing our findings and suggesting some future extensions of our results. In addition, an Appendix we have included the Concus-Finn comparison principle and its Corollary used in Sections 2C and 3A.

\section{Unbounded capillary surfaces}

In this section, we assume $\gamma_{1}+\gamma_{2} \neq \pi$ and aim to prove that

$$
u(x, y)=\Theta\left(\frac{1}{f_{1}(x)-f_{2}(x)}\right) \quad \text { as } x \rightarrow 0^{+},
$$

with as few restrictions on $f_{1}(x)$ and $f_{2}(x)$ as possible.

2A. Unboundedness of the capillary surface when $\gamma_{1}+\gamma_{2} \neq \pi$. We show that $u(x, y) \neq O(1)$. This is intuitively obvious from the remarkable result of Concus and Finn [1969], as a cusp can be considered as a corner with zero opening angle.

Lemma 2.1 (unboundedness of $u(x, y)$ when $\left.\gamma_{1}+\gamma_{2} \neq \pi\right)$. Let $u(x, y)$ be the solution of the boundary value problem (1-3)-(1-5).

If $\cos \gamma_{1}+\cos \gamma_{2}>0$, then $u(x, y)$ cannot be bounded from above.

If $\cos \gamma_{1}+\cos \gamma_{2}<0$, then $u(x, y)$ cannot be bounded from below.

Proof. Similar to the proof in [Concus and Finn 1969], we work by contradiction. First consider the case $\cos \gamma_{1}+\cos \gamma_{2}>0$, and assume there exists a constant $M>0$ such that $u(x, y)<M$ in $\Omega$. Integrate the PDE (1-3) in a subdomain $\Omega_{\epsilon}$ given by

$$
\Omega_{\epsilon}=\left\{(x, y): 0<x<\epsilon, f_{2}(x)<y<f_{1}(x)\right\} .
$$

By applying the divergence theorem and the boundary conditions (1-4) and (1-5), we obtain after some calculation the equation

$$
\begin{aligned}
& \int_{x=0}^{\epsilon} \int_{y=f_{2}(x)}^{f_{1}(x)} u d y d x \\
& =\int_{x=0}^{\epsilon}\left(\cos \gamma_{1} \sqrt{1+f_{1}^{\prime 2}}+\cos \gamma_{2} \sqrt{1+f_{2}^{\prime 2}}\right) d x+\left.\int_{y=f_{2}(\epsilon)}^{f_{1}(\epsilon)} \frac{u_{x}}{\sqrt{1+u_{x}^{2}+u_{y}^{2}}}\right|_{x=\epsilon} d x .
\end{aligned}
$$

The trick is to realize that the last term of (2-1) can be bounded from below, i.e.,

$$
\frac{u_{x}}{\sqrt{1+u_{x}^{2}+u_{y}^{2}}}>-1
$$


which implies

$$
\left.\int_{y=f_{2}(\epsilon)}^{f_{1}(\epsilon)} \frac{u_{x}}{\sqrt{1+u_{x}^{2}+u_{y}^{2}}}\right|_{x=\epsilon} d x>-\left(f_{1}(\epsilon)-f_{2}(\epsilon)\right) .
$$

We now apply the assumption $u(x, y)<M$ and the preceding inequality to (2-1) and obtain the inequality

$$
\begin{aligned}
\epsilon M \max _{0<x \leq \epsilon}\left(f_{1}(x)-f_{2}(x)\right)+\left(f_{1}(\epsilon)\right. & \left.-f_{2}(\epsilon)\right) \\
& >\int_{x=0}^{\epsilon}\left(\cos \gamma_{1} \sqrt{1+f_{1}^{\prime 2}}+\cos \gamma_{2} \sqrt{1+f_{2}^{\prime 2}}\right) d x .
\end{aligned}
$$

Dividing both sides by $\epsilon>0$ and taking the limit as $\epsilon$ approaches 0 gives

$$
\begin{aligned}
\lim _{\epsilon \rightarrow 0^{+}} M \max _{0<x \leq \epsilon}\left(f_{1}(x)-f_{2}(x)\right) & +\lim _{\epsilon \rightarrow 0^{+}} \frac{f_{1}(\epsilon)-f_{2}(\epsilon)}{\epsilon} \\
\geq & \lim _{\epsilon \rightarrow 0^{+}} \frac{\int_{x=0}^{\epsilon}\left(\cos \gamma_{1} \sqrt{1+f_{1}^{\prime 2}}+\cos \gamma_{2} \sqrt{1+f_{2}^{\prime 2}}\right) d x}{\epsilon} .
\end{aligned}
$$

Applying the definition of the derivative together with (1-2) then gives

$$
f_{1}^{\prime}(0)-f_{2}^{\prime}(0) \geq\left(\cos \gamma_{1} \sqrt{1+f_{1}^{\prime}(0)^{2}}+\cos \gamma_{2} \sqrt{1+f_{2}^{\prime}(0)^{2}}\right),
$$

which implies $0 \geq \cos \gamma_{1}+\cos \gamma_{2}$. Hence we obtain a contradiction. The proof for the case where $\cos \gamma_{1}+\cos \gamma_{2}<0$ can be constructed similarly.

Lemma 2.1 and Corollary A.1 together imply that $u(x, y)$ is unbounded at the cusp and bounded away from the cusp.

2B. Formal asymptotic expansion of the boundary value problem (1-3)-(1-5). The main idea is to consider an asymptotic expansion of the form

$$
v(x, y)=\frac{A}{f_{1}(x)-f_{2}(x)}+g(x, y) \frac{f_{1}^{\prime}(x)-f_{2}^{\prime}(x)}{f_{1}(x)-f_{2}(x)}+h(x, y) \frac{\left(f_{1}^{\prime}(x)-f_{2}^{\prime}(x)\right)^{2}}{f_{1}(x)-f_{2}(x)},
$$

where $g(x, y), h(x, y) \in O(1)$ as $x \rightarrow 0^{+}$. Recalling that $\lim _{x \rightarrow 0^{+}} f_{1}(x)=0$ and $\lim _{x \rightarrow 0^{+}} f_{2}(x)=0$, we have the first term significantly larger than the second term near the cusp. Also note that the leading order term is of the same order as the reciprocal of the distance between two boundaries measured in $\vec{y}$ direction.

The aim of this subsection is to find $g(x, y)$ and $h(x, y)$ such that (2-2) satisfies asymptotically the PDE (1-3) and the boundary conditions (1-4) and (1-5).

For simplicity of computation, we introduce coordinate variables $s$ and $t$ as follows:

$$
s:=x, \quad t:=\frac{2 y-\left(f_{1}(x)+f_{2}(x)\right)}{f_{1}(x)-f_{2}(x)} .
$$


We have chosen $t$ so that $y=f_{1}(x)$ when $t=1$, and $y=f_{2}(x)$ when $t=-1$.

Lemma 2.2 (first two terms of the formal asymptotic expansion). In (2-2), let $A=\cos \gamma_{1}+\cos \gamma_{2}$, and

$$
g(s, t)=-\sqrt{1-\left(\frac{\cos \gamma_{1}(t+1)+\cos \gamma_{2}(t-1)}{2}\right)^{2}}+C_{1}
$$

(where $C_{1}$ is an arbitrary constant), and $h(s, t)=0$. If $f_{1}(s)$ and $f_{2}(s)$ satisfy

$$
\begin{gathered}
f_{1}(s)-f_{2}(s)=o\left(f_{1}^{\prime}(s)-f_{2}^{\prime}(s)\right), \quad \frac{f_{1}^{\prime \prime}(s)-f_{2}^{\prime \prime}(s)}{f_{1}(s)-f_{2}(s)}=o\left(\frac{f_{1}^{\prime}(s)-f_{2}^{\prime}(s)}{\left(f_{1}(s)-f_{2}(s)\right)^{2}}\right), \\
\frac{f_{1}^{\prime \prime \prime}(s)-f_{2}^{\prime \prime \prime}(s)}{f_{1}^{\prime}(s)-f_{2}^{\prime}(s)}=o\left(\frac{1}{\left(f_{1}(s)-f_{2}(s)\right)^{2}}\right),
\end{gathered}
$$

as $s \rightarrow 0^{+}$, then

$$
\begin{gathered}
\left.\vec{v}_{1} \cdot T v\right|_{t=1}=\cos \gamma_{1}+o(1),\left.\quad \vec{v}_{2} \cdot T v\right|_{t=-1}=\cos \gamma_{2}+o(1), \\
\nabla \cdot T v-v=o\left(\frac{1}{f_{1}(s)-f_{2}(s)}\right)
\end{gathered}
$$

as $s \rightarrow 0^{+}$.

A tedious but straightforward calculation will verify this lemma. Instead of showing this calculation, we briefly explain here how the expressions for $A, g$, and $h$ in the statement of the lemma were deduced. We first let

$$
v(s, t)=\frac{A}{f_{1}(s)-f_{2}(s)}+g(t) \frac{f_{1}^{\prime}(s)-f_{2}^{\prime}(s)}{f_{1}(s)-f_{2}(s)} .
$$

(It is desirable - and, as it turns out, possible - to make the function $g$ depend only on $t$, so we will suppress the dependence of $g$ on $s$; the same applies to the function $h$.) After some lengthy calculations with assumptions (2-3) we obtain

$$
\begin{gathered}
\left.\vec{v}_{1} \cdot T v\right|_{t=1}=\frac{2 g^{\prime}(1)}{\sqrt{A^{2}+4 g^{\prime 2}(1)}}+o(1),\left.\quad \vec{v}_{2} \cdot T v\right|_{t=-1}=-\frac{2 g^{\prime}(-1)}{\sqrt{A^{2}+4 g^{\prime 2}(-1)}}+o(1), \\
\nabla \cdot T v-v=\left(\frac{4 g^{\prime \prime}(t) A^{2}}{\left(A^{2}+4 g^{\prime 2}(t)\right)^{3 / 2}}-A\right) \frac{1}{f_{1}(s)-f_{2}(s)}+o\left(\frac{1}{f_{1}(s)-f_{2}(s)}\right) .
\end{gathered}
$$

We now impose the desired equalities (2-4) and obtain a nonlinear ordinary differential equation of the first order in $g^{\prime}(t)$,

$$
\frac{4 g^{\prime \prime}(t) A^{2}}{\left(A^{2}+4 g^{\prime 2}(t)\right)^{3 / 2}}=A \quad \text { for }-1<t<1,
$$


with boundary conditions

$$
\frac{2 g^{\prime}(1)}{\sqrt{A^{2}+4 g^{\prime 2}(1)}}=\cos \gamma_{1}, \quad-\frac{2 g^{\prime}(-1)}{\sqrt{A^{2}+4 g^{\prime 2}(-1)}}=\cos \gamma_{2} .
$$

Though there are two boundary conditions for this first-order ODE, note that $A$ is an indeterminate constant. Both $g^{\prime}(t)$ and $A$ are determined by first integrating (2-5) udner the boundary conditions (2-6). One essential observation from this derivation is that the coefficient $A$ of the leading-order term was found together with that of the second-order term, $g(t)$. In fact this pattern continues; the constant on the secondorder term $C_{1}$ will be determined (it vanishes) at the same time as the third-order term of the formal asymptotic expansion is found.

Lemma 2.3 (first three terms of the formal asymptotic expansion). In (2-2), let $A=\cos \gamma_{1}+\cos \gamma_{2}$,

$$
g(t)=-\sqrt{1-\left(\frac{\cos \gamma_{1}(t+1)+\cos \gamma_{2}(t-1)}{2}\right)^{2}},
$$

and

$$
h(t)=-\frac{A}{4}\left(\delta t+\frac{t^{2}}{2}\right)+\frac{1-\alpha}{2 A} g(t)^{2}+C_{2},
$$

where $C_{2}$ is an arbitrary constant. If $f_{1}(s)$ and $f_{2}(s)$ satisfy the conditions

$$
\begin{aligned}
f_{1}^{\prime}(s) & >f_{2}^{\prime}(s) \quad \text { for } s>0, \\
f_{1}(s)-f_{2}(s) & =o\left(f_{1}^{\prime}(s)-f_{2}^{\prime}(s)\right), \\
\frac{f_{1}^{\prime \prime}(s)-f_{2}^{\prime \prime}(s)}{f_{1}(s)-f_{2}(s)} & =\alpha \frac{\left(f_{1}^{\prime}(s)-f_{2}^{\prime}(s)\right)^{2}}{\left(f_{1}(s)-f_{2}(s)\right)^{2}}+o\left(\frac{\left(f_{1}^{\prime}(s)-f_{2}^{\prime}(s)\right)^{2}}{\left(f_{1}(s)-f_{2}(s)\right)^{2}}\right), \\
\frac{f_{1}^{\prime \prime \prime}(s)-f_{2}^{\prime \prime \prime}(s)}{f_{1}^{\prime}(s)-f_{2}^{\prime}(s)} & =O\left(\frac{\left(f_{1}^{\prime}(s)-f_{2}^{\prime}(s)\right)^{2}}{\left(f_{1}(s)-f_{2}(s)\right)^{2}}\right),
\end{aligned}
$$$$
\text { as } s \rightarrow 0^{+} \text {, where } \alpha, \delta \in \mathbb{R} \text {, then }
$$$$
\left.\vec{v}_{1} \cdot T v\right|_{t=1}=\cos \gamma_{1}+o\left(f_{1}^{\prime}(s)-f_{2}^{\prime}(s)\right),\left.\quad \vec{v}_{2} \cdot T v\right|_{t=-1}=\cos \gamma_{2}+o\left(f_{1}^{\prime}(s)-f_{2}^{\prime}(s)\right),
$$$$
\nabla \cdot T v-v=o\left(\frac{f_{1}^{\prime}(s)-f_{2}^{\prime}(s)}{f_{1}(s)-f_{2}(s)}\right)
$$

as $s \rightarrow 0^{+}$. 
Again, a long tedious calculation will prove this lemma. We followed similar steps to determine $h(t)$, although solving the differential equation for $h(t)$ was not nearly as straightforward as for $g(t)$. The constant $C_{1}$ was determined to be 0 when $h(t)$ was determined and a new unknown constant $C_{2}$ appeared in the third-order term.

Comparing assumptions (2-3) with assumptions (2-8)-(2-12), we can see that the restrictions on $f_{1}$ and $f_{2}$ increase as the number of terms in the formal asymptotic expansion increases from two terms to three terms. Although these assumptions are not proven to be necessary conditions for these lemmas to hold, it is our suspicion that as the number of the terms in the asymptotic expansion increases, the restrictions on $f_{1}$ and $f_{2}$ do become more strict.

2C. Asymptotic behavior of the capillary surface. The main result of Section 2 is stated and proven in this subsection. We first show that the asymptotic growth order of the solution is the same order as the reciprocal of the distance between two arcs forming a cusp.

Theorem 2.1 (growth order of $u(x, y)$ ). Let $u(x, y)$ be the solution of the boundary value problem (1-3)-(1-5). If $f_{1}(s)$ and $f_{2}(s)$ satisfy the conditions (2-3) and $\left|\cos \gamma_{1}\right| \neq 1$ and $\left|\cos \gamma_{2}\right| \neq 1$, then there exist positive constants $s_{0}, k_{1}$ and $k_{2}$ such that

$$
k_{2}\left(\frac{1}{f_{1}(s)-f_{2}(s)}\right)<|u(s, t)|<k_{1}\left(\frac{1}{f_{1}(s)-f_{2}(s)}\right), \quad \text { for } s<s_{0} .
$$

Proof. The main idea of our proof is to construct a supersolution and a subsolution by modifying the formal asymptotic expansion given in Lemma 2.2. We prove these modified equations are in fact supersolution and subsolution by applying the Concus-Finn comparison principle (Theorem A.1). Let

$$
v\left(s, t ; K_{1}, K_{2}\right)=\frac{A\left(K_{1}\right)}{f_{1}(s)-f_{2}(s)}+g\left(t ; K_{1}\right) \frac{f_{1}^{\prime}(s)-f_{2}^{\prime}(s)}{f_{1}(s)-f_{2}(s)}+K_{2},
$$

where

$$
A\left(K_{1}\right)=\cos \gamma_{1}+\cos \gamma_{2}+K_{1},
$$

$$
g\left(t ; K_{1}\right)=-\frac{A}{A-\frac{1}{3} K_{1}} \sqrt{1-\left(\frac{\cos \gamma_{1}(t+1)+\cos \gamma_{2}(t-1)}{2}-\frac{K_{1}}{6} t\right)^{2}} ;
$$

here we choose $K_{1}$ and $K_{2}$ appropriately to construct the supersolution and the subsolution. The trick of this proof is to realize that $A$ and $g(t)$, the first and second terms of the formal asymptotic expansion, need to be modified to obtain a supersolution and a subsolution. We first impose the following conditions on $K_{1}$ 
so that the quantities in (2-14) behave reasonably:

$$
\begin{aligned}
& \left|K_{1}\right|<\left|\cos \gamma_{1}+\cos \gamma_{2}\right|, \\
& \left|K_{1}\right|<6\left(1-\left|\cos \gamma_{1}\right|\right), \\
& \left|K_{1}\right|<6\left(1-\left|\cos \gamma_{2}\right|\right) .
\end{aligned}
$$

We restrict the choice of $K_{1}$ so that the sign of $A\left(K_{1}\right)$ only depends on the sign of $\cos \gamma_{1}+\cos \gamma_{2}$. Also, if $K_{1}$ is chosen to satisfy (2-15)-(2-17), then $g\left(t, K_{1}\right)$ is real and bounded. After some calculations assuming (2-3), we obtain

$$
\begin{gathered}
\left.\vec{v}_{1} \cdot T v\right|_{t=1}=\cos \gamma_{1}+\frac{1}{3} K_{1}+o(1),\left.\quad \vec{v}_{2} \cdot T v\right|_{t=-1}=\cos \gamma_{2}+\frac{1}{3} K_{1}+o(1), \\
\nabla \cdot T v-v=-\frac{1}{3} K_{1} \frac{1}{f_{1}(s)-f_{2}(s)}-K_{2}+o\left(\frac{f_{1}^{\prime}(s)-f_{2}^{\prime}(s)}{f_{1}(s)-f_{2}(s)}\right)
\end{gathered}
$$

as $s \rightarrow 0^{+}$. The essential observation in this step of the proof is that the expressions in (2-18) do not depend on $K_{2}$ including the "small o" terms. Similarly, (2-19) has $K_{2}$ dependence only at the second term and not in the "small o" term.

We now construct a function $v^{+}$that satisfies inequalities (A-1)-(A-4) in the Appendix, and is therefore a supersolution. We denote the associated constants by $K_{1}^{+}$and $K_{2}^{+}$; i.e., $v^{+}=v\left(s, t ; K_{1}^{+}, K_{2}^{+}\right)$. Firstly, $K_{1}^{+}$are chosen to be a small enough positive real number so as to satisfy $(2-15)-(2-17)$. Then we choose a constant $s_{0}^{+}>0$ so that for all $s<s_{0}^{+}$the inequalities

$$
\begin{gathered}
\left.\vec{v}_{1} \cdot T v^{+}\right|_{t=1}-\cos \gamma_{1}>0,\left.\quad \vec{v}_{2} \cdot T v^{+}\right|_{t=-1}-\cos \gamma_{2}>0, \\
\nabla \cdot T v^{+}-v^{+}+K_{2}^{+}<0 .
\end{gathered}
$$

are satisfied. Based on our previous observation we note that the choice of $s_{0}^{+}$is independent of $K_{2}^{+}$. Let $\Omega_{0}^{+}$be the subdomain of $\Omega$ such that $s<s_{0}^{+}$. By adding a restriction on $K_{2}^{+}$to be a positive real number, it follows from (2-21) that

$$
\nabla \cdot T v^{+}-v^{+}<0 \quad \text { in } \Omega_{0}^{+} .
$$

Note that $v^{+}$now satisfies conditions (A-1)-(A-3) of the Concus-Finn comparison principle (Theorem A.1). It remains to choose $K_{2}^{+}$so as to satisfy condition (A-4). According to Corollary A.1, $u(s, t)$ is bounded at $s=s_{0}^{+}$. Hence there exists $K_{2}^{+}$ such that

$$
v^{+}>u \quad \text { on } s=s_{0}^{+} \text {. }
$$

Thus by Theorem A.1 we have shown that there exists $\Omega_{0}^{+}, K_{1}^{+}, K_{2}^{+}$such that

$$
v^{+}\left(s, t ; K_{1}^{+}, K_{2}^{+}\right)>u(s, t) \text { in } \Omega_{0}^{+} .
$$


Similarly we can construct a subsolution $v^{-}\left(s, t ; K_{1}^{-}, K_{2}^{-}\right)$such that

$$
v^{-}\left(s, t ; K_{1}^{-}, k_{2}^{-}\right)<u(s, t) \text { in } \Omega_{0}^{-} .
$$

Hence in $\Omega_{0}^{+} \cap \Omega_{0}^{-}$we have $v^{-}<u<v^{+}$, i.e.,

$$
\frac{A\left(K_{1}^{-}\right)}{f_{1}(s)-f_{2}(s)}+g\left(t ; K_{1}^{-}\right) \frac{f_{1}^{\prime}(s)-f_{2}^{\prime}(s)}{f_{1}(s)-f_{2}(s)}+K_{2}^{-}<u
$$

and

$$
u<\frac{A\left(K_{1}^{+}\right)}{f_{1}(s)-f_{2}(s)}+g\left(t ; K_{1}^{+}\right) \frac{f_{1}^{\prime}(s)-f_{2}^{\prime}(s)}{f_{1}(s)-f_{2}(s)}+K_{2}^{+} .
$$

Since $K_{1}^{+}$and $K_{1}^{-}$were chosen to satisfy $(2-15), A\left(K_{1}^{+}\right)$and $A\left(K_{1}^{-}\right)$have the same sign. Without loss of generality assume $A\left(K_{1}^{+}\right)>0$. Let

$$
\begin{aligned}
& m_{1}(s)=A\left(K_{1}^{+}\right)+\left(\max _{-1<t<1}\left\{g\left(t ; K_{1}^{+}\right)\left(f_{1}^{\prime}(s)-f_{2}^{\prime}(s)\right)\right\}+K_{2}^{+}\left(f_{1}(s)-f_{2}(s)\right)\right), \\
& m_{2}(s)=A\left(K_{1}^{-}\right)+\left(\min _{-1<t<1}\left\{g\left(t ; K_{1}^{-}\right)\left(f_{1}^{\prime}(s)-f_{2}^{\prime}(s)\right)\right\}+K_{2}^{-}\left(f_{1}(s)-f_{2}(s)\right)\right) .
\end{aligned}
$$

Since $f_{1}^{\prime}(s)-f_{2}^{\prime}(s)$ and $f_{1}(s)-f_{2}(s)$ are $o(1)$ and continuous, there exists $s_{0}>0$ so that $m_{1}(s), m_{2}(s)>0$ for $s<s_{0}$. By choosing

$$
k_{1}=\max _{0<s<s_{0}} m_{1}(s), \quad k_{2}=\min _{0<s<s_{0}} m_{2}(s),
$$

we obtain (2-13).

Note that the proof holds for arbitrarily small $\left|K_{1}^{ \pm}\right|$. Hence it is natural to guess that $\left(\cos \gamma_{1}+\cos \gamma_{2}\right) /\left(f_{1}(s)-f_{2}(s)\right)$ is the correct leading-order term of the asymptotic expansion. We now show that the leading-order term of the formal asymptotic expansion is in fact the first-order term of the asymptotic expansion of $u(s, t)$.

Theorem 2.2 (leading-order behavior of $u(x, y)$ ). Let $u(x, y)$ be the solution of the boundary value problem (1-3)-(1-5). Assume that $f_{1}(s)$ and $f_{2}(s)$ satisfy the conditions (2-8)-(2-12). Then

$$
u(s, t)=\frac{\cos \gamma_{1}+\cos \gamma_{2}}{f_{1}(s)-f_{2}(s)}+O\left(\frac{f_{1}^{\prime}(s)-f_{2}^{\prime}(s)}{f_{1}(s)-f_{2}(s)}\right) \quad \text { as } s \rightarrow 0^{+} .
$$

Proof. We let

$$
\begin{aligned}
v\left(s, t ; K_{3}, K_{4}, K_{5}\right)=\frac{A}{f_{1}(s)-f_{2}(s)}+g\left(t, K_{3}\right) & \frac{f_{1}^{\prime}(s)-f_{2}^{\prime}(s)}{f_{1}(s)-f_{2}(s)} \\
& +h\left(t ; K_{4}\right) \frac{\left(f_{1}^{\prime}(s)-f_{2}^{\prime}(s)\right)^{2}}{f_{1}(s)-f_{2}(s)}+K_{5},
\end{aligned}
$$


where

$A=\cos \gamma_{1}+\cos \gamma_{2}$,

$g\left(t ; K_{3}\right)=-\sqrt{1-\left(\frac{\cos \gamma_{1}(t+1)+\cos \gamma_{2}(t-1)}{2}\right)^{2}}+K_{3}$,

$h\left(t ; K_{4}\right)=-\frac{A}{4}\left(\delta t+\frac{t^{2}}{2}\right)+\frac{1-\alpha}{2 A}\left\{1-\left(\frac{\cos \gamma_{1}(t+1)+\cos \gamma_{2}(t-1)}{2}\right)^{2}\right\}+\frac{K_{4}}{2} t^{2}$.

Unlike the proof of Theorem 2.1, we can choose $K_{3}$ and $K_{4}$ as any real numbers. After some calculations assuming (2-8)-(2-12), we obtain

$$
\left.\vec{v}_{1} \cdot T v\right|_{t=1}=\cos \gamma_{1}+K_{4} \frac{\left(f_{1}^{\prime}(s)-f_{2}^{\prime}(s)\right)}{\left(A^{2}+4\left(g^{\prime}(t)\right)^{2}\right)^{3 / 2}}+o\left(f_{1}^{\prime}(s)-f_{2}^{\prime}(s)\right),
$$

$$
\left.\vec{v}_{2} \cdot T v\right|_{t=-1}=\cos \gamma_{2}+K_{4} \frac{\left(f_{1}^{\prime}(s)-f_{2}^{\prime}(s)\right)}{\left(A^{2}+4\left(g^{\prime}(t)\right)^{2}\right)^{3 / 2}}+o\left(f_{1}^{\prime}(s)-f_{2}^{\prime}(s)\right),
$$

$\nabla \cdot T v-v=\left\{\left(-\frac{12 g^{\prime}(t) t}{A^{2}+4\left(g^{\prime}(t)\right)^{2}}+\frac{4 A^{2}}{\left(A^{2}+4\left(g^{\prime}(t)\right)^{2}\right)^{3 / 2}}\right) K_{4}-K_{3}\right\} \frac{f_{1}^{\prime}(s)-f_{2}^{\prime}(s)}{f_{1}(s)-f_{2}(s)}$

$$
-K_{5}+o\left(\frac{f_{1}^{\prime}(s)-f_{2}^{\prime}(s)}{f_{1}(s)-f_{2}(s)}\right),
$$

as $s \rightarrow 0^{+}$.

We now construct a supersolution. Let $v^{+}$denote the supersolution, with associate constants $K_{3}^{+}, K_{4}^{+}, K_{5}^{+}$; i.e., $v^{+}=v\left(s, t ; K_{3}^{+}, K_{4}^{+}, K_{5}^{+}\right)$. We first choose the positive constant $K_{4}^{+}$arbitrarily. Then we choose $K_{3}^{+}$big enough so that

$$
\left\{\left(-\frac{12 g^{\prime}(t) t}{A^{2}+4\left(g^{\prime}(t)\right)^{2}}+\frac{4 A^{2}}{\left(A^{2}+4\left(g^{\prime}(t)\right)^{2}\right)^{3 / 2}}\right) K_{4}^{+}-K_{3}^{+}\right\}<0 \quad \text { for }-1<t<1 .
$$

We now choose $s_{2}^{+}>0$ so that

$$
\left.\vec{v}_{1} \cdot T v\right|_{t=1}-\cos \gamma_{1}>0,\left.\quad \vec{v}_{2} \cdot T v\right|_{t=-1}-\cos \gamma_{2}>0, \quad \nabla \cdot T v-v+K_{5}^{+}<0
$$

for $0<s<s_{2}^{+}$. Let $\Omega_{2}^{+}$be the subdomain of $\Omega$ such that $s<s_{2}^{+}$. By Corollary A.1, we know that $u\left(s_{2}^{+}, t\right)$ is bounded; hence there exists a large enough positive constant $K_{5}^{+}$so that

$$
v^{+}>u \quad \text { on } s=s_{2}^{+} \text {. }
$$

Thus by the Concus-Finn comparison principle (Theorem A.1) we have

$$
v^{+}>u \text { in } \Omega_{2}^{+} \text {. }
$$


Similarly we can construct a subsolution $v^{-}$by choosing suitable $K_{3}^{-}, K_{4}^{-}, K_{5}^{-}$ and $s_{2}^{-}$. Thus we can bound the solution $u(s, t)$ by $v^{-}$and $v^{+}$; i.e.,

$$
v^{-}<u<v^{+} \text {in } \Omega_{2}^{+} \cap \Omega_{2}^{-},
$$

and (2-23) holds.

From this section, we conclude that the height of a capillary surface near a cusp is proportional to the reciprocal of the distance between the two arcs forming the cusp, assuming these arcs satisfy (2-3).

2D. Examples of cusp domains. In the previous subsection, we have shown the behavior of the capillary surface near a cusp under certain assumptions $f_{1}(x)$ and $f_{2}(x)$ giving the shape of the boundaries. Those assumptions, expressed by (2-3) or (2-8)-(2-12), are left in these forms in order to make the theorem as general as possible. On the other hand, it is hard to grasp what kind of cusps are allowed or not. In this subsection, we will show through examples when the theorem is applicable and when it is not.

It is easy to show that if the difference between $f_{1}$ and $f_{2}$ can be written in the following form, these functions satisfy (2-8)-(2-10):

$$
f_{1}(x)-f_{2}(x)=c x^{a_{0}} \exp \left(\sum_{i=1}^{\infty} a_{i} x^{b_{i}}\right),
$$

where $c>0, a_{1}<0, b_{1}<0, b_{i+1}>b_{i}$. An alternative way to write this is

$$
f_{1}(x)-f_{2}(x)=\exp \left(\int_{c}^{x} \frac{\sum_{i=0}^{\infty} \tilde{a}_{i} \tilde{b}^{\tilde{b}_{i}}}{\sum_{i=0}^{\infty} a_{i} \zeta^{b_{i}}} d \zeta\right),
$$

where $c>0, b_{0}-\tilde{b}_{0} \geq 1, b_{i+1}>b_{i}, a_{0}>0$ and $\tilde{a}_{0}>0$. As (2-8)-(2-10) are stricter requirements for $f_{1}(x)$ and $f_{2}(x)$ than (2-3), if $f_{1}(x)-f_{2}(x)$ can be written as (2-27) or (2-28), then $f_{1}$ and $f_{2}$ satisfy (2-3).

Note that (2-11) and (2-12) can be interpreted as saying that some osculating cusps (cusps with boundaries tangent to second order) are not allowed, and Equation (2-7) can be interpreted as saying that infinitely oscillating cusp boundaries are not allowed.

Example 1 (fractional power cusp). We now consider a cusp that can be analyzed through the result of Scholz. Consider (2-28) and let $b_{0}>1, \tilde{a}_{i}=a_{i} b_{i}$ and $\tilde{b}_{i}=$ $b_{i}-1$. Then we have

$$
f_{1}(x)-f_{2}(x)=\tilde{c} \sum_{i=0}^{\infty} a_{i} x^{b_{i}}
$$



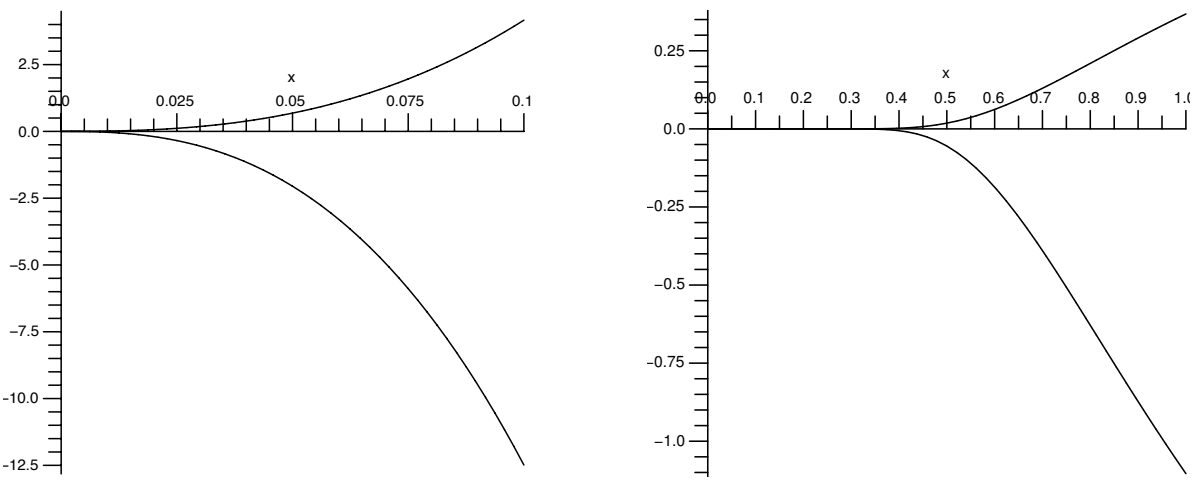

Figure 2. Left: fractional power cusp (Example 1). Right: exponential cusp (Example 2). In both cases, $p=1$ and $q=-3$.

To be more specific, we consider the cusp boundaries

$$
f_{1}(x)=p\left(x^{5 / 2}+x^{3}\right), \quad f_{2}(x)=q\left(x^{5 / 2}+x^{3}\right),
$$

with constants $p>q$ (see Figure 2, left). According to Theorem 2.2, we obtain the asymptotic expansion

$$
\begin{aligned}
u(x, y) & =\frac{\cos \gamma_{1}+\cos \gamma_{2}}{(p-q)\left(x^{5 / 2}+x^{3}\right)}+O\left(x^{-1}\right) \\
& =\frac{\cos \gamma_{1}+\cos \gamma_{2}}{p-q}\left(\frac{1}{x^{5 / 2}}-\frac{1}{x^{2}}+\frac{1}{x^{3 / 2}}\right)+O\left(x^{-1}\right)
\end{aligned}
$$

as $x \rightarrow 0^{+}$. We note that this result is consistent with that of Scholz. It is noteworthy that by finding the first order term of our asymptotic expansion we find the first three terms of the asymptotic series solution in power series.

Example 2 (exponential cusp). We now consider cusps to which the results of Scholz do not apply. Equation (2-27) implies that $f_{1}(x)$ and $f_{2}(x)$ can contain exponential terms. We now consider a very sharp cusp, an "exponential cusp", where

$$
f_{1}(x)=p e^{-1 / x^{2}}, \quad f_{2}(x)=q e^{-1 / x^{2}} .
$$

with constants $p>q$ (see Figure 2, right). According to Theorem 2.2, we obtain the asymptotic expansion

$$
u(x, y)=\frac{\cos \gamma_{1}+\cos \gamma_{2}}{p-q} e^{1 / x^{2}}+O\left(x^{-3}\right) \quad \text { as } x \rightarrow 0^{+} .
$$

This example shows that our result has extended the result of Scholz on the leading order behavior of a capillary surface in a cusp domain. 


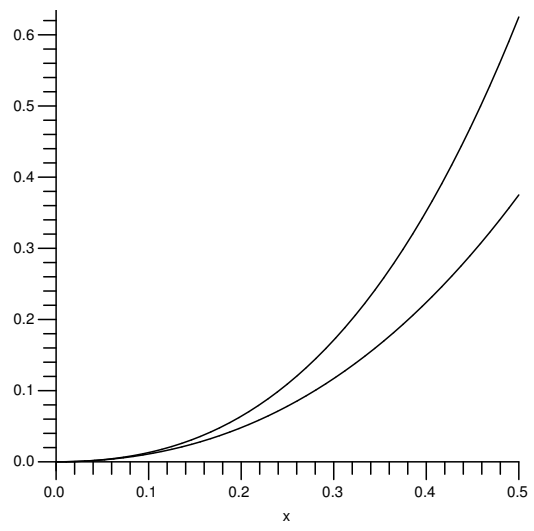

Figure 3. Osculatory cusp $(p=3, q=1)$.

Example 3 (osculatory cusp). We now consider a case where Theorem 2.2 cannot be applied. Consider the cusp boundaries

$$
f_{1}(x)=x^{2}+p x^{3}, \quad f_{2}(x)=x^{2}+q x^{3},
$$

with constants $p>q$ (see Figure 3).

These functions do not satisfy (2-11)-(2-12); hence Theorem 2.2 does not apply. On the other hand, if $\left|\cos \gamma_{1}\right| \neq 1$ and $\left|\cos \gamma_{2}\right| \neq 1$, Theorem 2.1 applies, as this $f_{1}$ and $f_{2}$ satisfy (2-3). Hence even the case of the osculating cusp, we have shown that the height of the capillary surface rises as the same order as the reciprocal of the distance of two arcs forming a cusp, i.e.,

$$
u(x, y)=\Theta\left(\frac{1}{x^{3}}\right) .
$$

As the two functions $f_{1}$ and $f_{2}$ forming a cusp only appear as $\left(f_{1}(x)-f_{2}(x)\right)$ or $\left(f_{1}^{\prime}(x)-f_{2}^{\prime}(x)\right)$ in the asymptotic expansion (2-2), it is not immediately obvious as to why we cannot conduct the asymptotic analysis of this problem similarly to the case where $f_{1}(x)=p x^{3}, f_{2}(x)=q x^{3}$. However, the difference in asymptotic order between $f_{1}(x)-f_{2}(x)$ on the one hand and $f_{1}(x)$ or $f_{2}(x)$ on the other becomes crucial in calculating the asymptotic relations (2-24)-(2-26) of the boundary conditions and the PDE. For example, for the calculation of (2-24), since

$$
\overrightarrow{v_{1}}=\frac{\left(-f_{1}^{\prime}(x), 1\right)}{\sqrt{1+\left(f_{1}^{\prime}(x)\right)^{2}}},
$$

the function $f_{1}(x)$ appears without subtracting $f_{2}(x)$. As a result, the asymptotic relation (2-24) does not hold for the case of osculatory cusp. Thus for the osculatory cusps, we cannot use the asymptotic expansion (2-2) to prove the leading order behavior. 


\section{Bounded capillary surfaces}

In this section we assume $\gamma_{1}+\gamma_{2}=\pi$ and prove that $u(x, y)$ is bounded.

\section{A. Proof of the boundedness of the capillary surface when $\gamma_{1}+\gamma_{2}=\pi$.}

Theorem 3.1 (boundedness of $u(x, y)$ when $\left.\gamma_{1}+\gamma_{2}=\pi\right)$. Let $u(x, y)$ be the solution of the boundary value problem (1-3)-(1-4) with $\gamma_{1}=\gamma$ and $\gamma_{2}=\pi-\gamma$. If the boundaries $\partial \Omega_{1}$ and $\partial \Omega_{2}$ have finite curvatures in the neighborhood of the cusp, in other words, if there exists $\epsilon_{o}$ such that

$$
f_{1}(x), f_{2}(x) \in C^{2}\left(\left[0, \epsilon_{o}\right]\right),
$$

then $u(x, y)$ is bounded.

Proof. It follows immediately from Corollary A.1 that $u(x, y)$ is bounded in the domain away from the origin. Hence our problem reduces to show that $u(x, y)$ is bounded in the neighborhood of the origin.

First we show that $u(x, y)$ is bounded above at the origin by using the ConcusFinn comparison principle (Theorem A.1). In order to apply Theorem A.1, we need to construct a surface that satisfies (A-1)-(A-4). The most difficult part of this proof is to construct a surface that satisfies both (A-2) and (A-3). Our unique idea is to construct a surface that satisfies (1-4) exactly hence (A-2) and also satisfies (A-3). Such surface can be constructed by a surface with contour lines parallel to the boundary $\partial \Omega_{1}$. In other words by letting the height of the surface only depends on the distance from the boundary $\partial \Omega_{1}$, we can easily construct a surface with exact constant contact angle $\gamma$ on this boundary. We choose a surface so that the height and the mean curvature is bounded so that Inequalities (A-1) and (A-4) can easily be satisfied by shifting this surface upwards.

We now translate the above statement to the precise language of mathematics. Without loss of generality we assume $0 \leq \gamma \leq \pi / 2$. First we define a coordinate system such that the one family of the coordinate curves is parallel curves of the boundary $\partial \Omega_{1}$ and another family of the coordinate curves is lines perpendicular to the boundary $\partial \Omega_{1}$. Let $s$ and $t$ be new coordinate variables defined implicitly as the following (note that $s$ here has different meaning from $s$ used in Section 2):

$$
(x, y)=\left(s, f_{1}(s)\right)-t \vec{v}_{1}(s),
$$

where $\vec{v}_{1}(s)$ is the exterior unit normal vector of the boundary $\partial \Omega_{1}$ at $\left(s, f_{1}(s)\right)$. More explicitly, the coordinate variables of Cartesian coordinate system $x$ and $y$ can be written using the new coordinate variables $s$ and $t$ as follows:

$$
x=s+t \frac{f_{1}^{\prime}(s)}{\sqrt{1+\left(f_{1}^{\prime}(s)\right)^{2}}}, \quad y=f_{1}(s)-t \frac{1}{\sqrt{1+\left(f_{1}^{\prime}(s)\right)^{2}}} .
$$



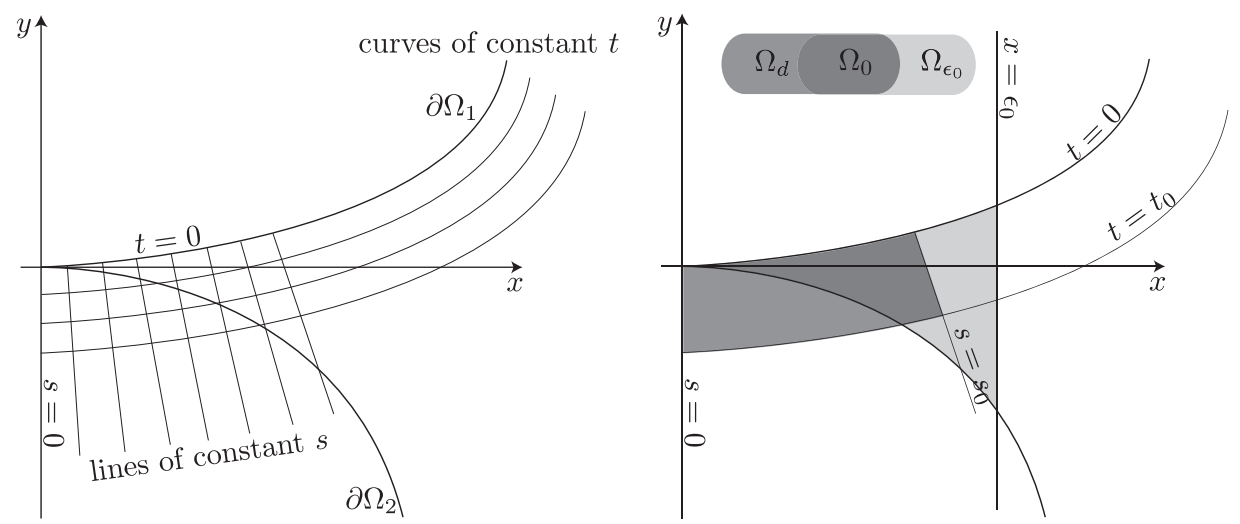

Figure 4. Left: coordinate lines of the $s-t$ coordinate system.

Right: the domain $\Omega_{0}$.

The variable $t$ can be interpreted as the distance of the point from the boundary $\partial \Omega_{1}$. The coordinate curves are sketched in Figure 4, left.

The Jacobian of (3-3) is calculated to be

$$
\frac{\partial(x, y)}{\partial(s, t)}=\frac{f_{1}^{\prime}(s)^{2}-1}{\sqrt{1+\left(f_{1}^{\prime}(s)\right)^{2}}}\left(1+t \frac{f_{1}^{\prime \prime}(s)}{\left(1+\left(f_{1}^{\prime}(s)\right)^{2}\right)^{3 / 2}}\right) .
$$

This gives that the point $(x, y)$ in the Cartesian coordinate system can be specified uniquely by the new coordinate variables $(s, t)$ defined by (3-3) if both

$$
f_{1}^{\prime}(s)^{2}-1 \neq 0
$$

and

$$
1+t \frac{f_{1}^{\prime \prime}(s)}{\left(1+\left(f_{1}^{\prime}(s)\right)^{2}\right)^{3 / 2}} \neq 0 .
$$

Since $f_{1}(s) \in C^{2}\left(\left[0, \epsilon_{o}\right]\right)$ and $\lim _{s \rightarrow 0^{+}} f_{1}(s)=0$, there exists $0<s_{0} \leq \epsilon_{0}$ so that (3-4) is satisfied for all $s \in\left[0, s_{0}\right]$. Also due to the smoothness of $f_{1}(s)$, we can find $t_{0}>0$ such that (3-5) holds for all $t \in\left[0, t_{0}\right]$ in $s \in\left[0, s_{0}\right]$. That is to say, the coordinate system defined in (3-3) is valid in the domain

$$
\Omega_{d}:=\left\{\left(s, f_{1}(s)\right)-t \vec{v}_{1}(s) \in \mathbb{R}^{2}: 0 \leq s \leq s_{0}, 0 \leq t \leq t_{0}\right\} .
$$

Then we choose the subdomain

$$
\Omega_{0}:=\Omega_{d} \cap \Omega_{\epsilon_{0}},
$$

where $\Omega_{\epsilon_{0}}:=\left\{(x, y) \in \mathbb{R}^{2}: 0<x<\epsilon_{0}, f_{2}(x)<y<f_{1}(x)\right\}$, as depicted in Figure 4, right. Since $\bar{\Omega}_{0}$ contains the cusp at the origin, finding an upper bound for the surface $u$ in domain $\Omega_{0}$ by using Theorem A.1 would prove that the capillary surface 

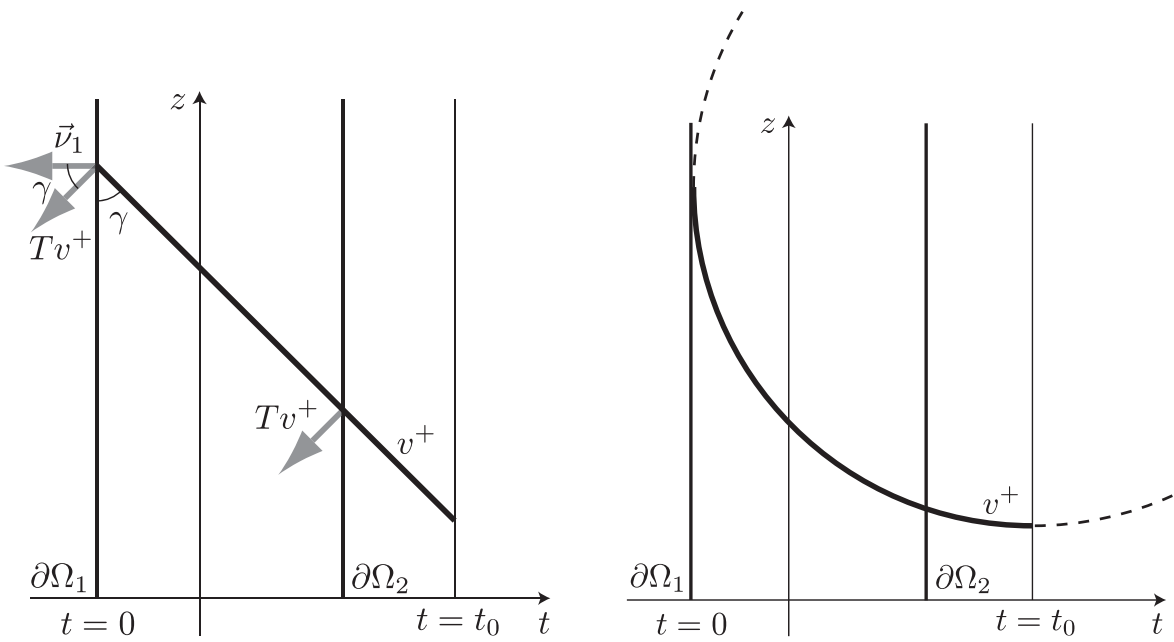

Figure 5. Cross section of a surface $v^{+}(s, t)$ on the line of constant $s$ : Choice of function $g(t)$ for $\gamma \neq 0$ (left) and for $\gamma=0$ (right).

is bounded above at the cusp. Using the parameters $t$ and $s$, we now construct a surface $v^{+}(s, t)$ in $\Omega_{0}$, with components $(x, y, z)$, as follows:

$x(s, t)=s+t \frac{f_{1}^{\prime}(s)}{\sqrt{1+\left(f_{1}^{\prime}(s)\right)^{2}}}, \quad y(s, t)=f_{1}(s)-t \frac{1}{\sqrt{1+\left(f_{1}^{\prime}(s)\right)^{2}}}, \quad z(s, t)=g(t)$.

The choice of the height function $g(t)$ depends on the contact angle $\gamma$. In our opinion, the simplest choice such that the surface $v^{+}$satisfies (1-4) exactly and also satisfies (A-3) is

$$
g(t)= \begin{cases}-\cot \gamma t+K & \text { for } \gamma \neq 0, \\ -\sqrt{t_{0}^{2}-\left(t-t_{0}\right)^{2}}+K & \text { for } \gamma=0,\end{cases}
$$

where $K$ is a constant that we will specify later. The cross section of this surface on a line of constant $s$ is depicted in Figure 5, left.

The surface $v^{+}(s, t)$ can be sketched as in Figure 6. For example, if the curve $\partial \Omega_{1}$ is a part of a circle, then the surface $v^{+}(s, t)$ for the case $\gamma \neq 0$ becomes a part of a cone, and for the case $\gamma=0$ it becomes a part of a torus.

We now verify that the surface $v^{+}(s, t)$ satisfies (1-4) exactly and also satisfies (A-3). We first consider the case $\gamma \neq 0$, as the vector $T v^{+}$can be interpreted as a unit downwards vector of the surface $v^{+}$, it follows immediately from Figure 5 (left) that $T v^{+}(s, t)$ can be written as

$$
T v^{+}=\cos \gamma \vec{v}_{1}-\sin \gamma \hat{z}
$$



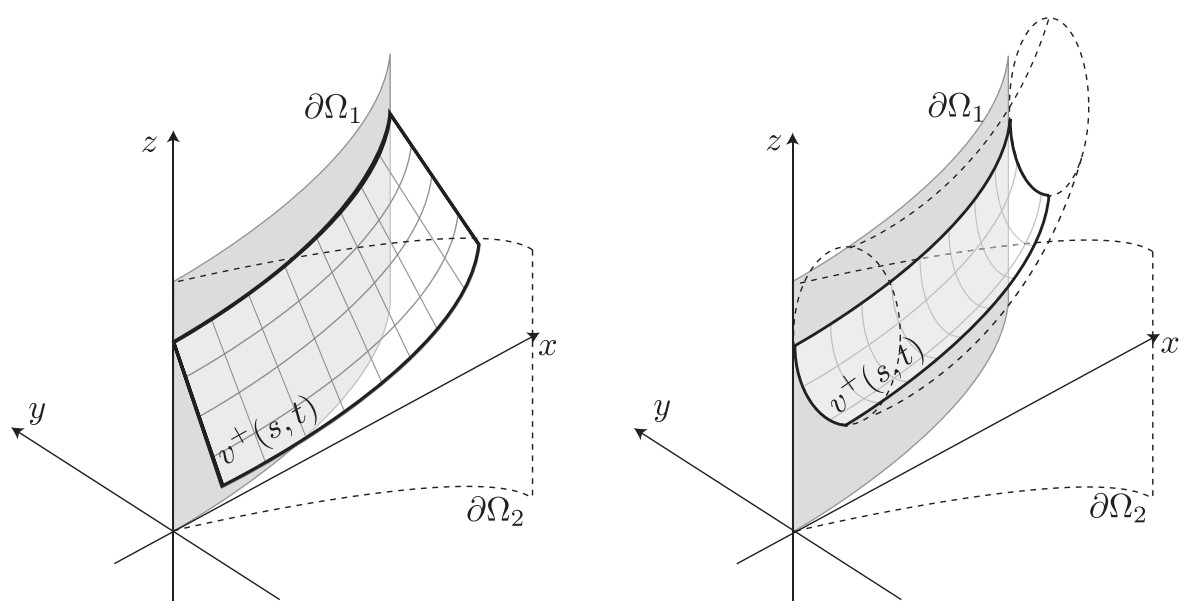

Figure 6. Sketch of the surface $v^{+}(s, t)$ for $\gamma \neq 0$ (left) and for $\gamma=0$ (right).

where $\hat{z}$ is a unit vector in $z$ direction. Noting that the vector $\vec{v}_{1}$ is orthogonal to $\hat{z}$, we obtain that (1-4) is satisfied exactly by the surface $v^{+}(s, t)$, i.e.,

$$
\vec{v}_{1} \cdot T v^{+}=\cos \gamma \quad \text { on } \partial \Omega_{1} \cap \partial \Omega_{0} .
$$

We now verify that the surface $v^{+}(s, t)$ satisfies Inequality (A-3). By noticing $\vec{v}_{2}$ and $\hat{z}$ are orthogonal and both $\vec{v}_{1}$ and $\vec{v}_{2}$ are unit vectors, we obtain the inequality

$$
\vec{v}_{2} \cdot T v^{+}=\cos \gamma \vec{v}_{1} \cdot \vec{v}_{2},>-\cos \gamma,=\cos (\pi-\gamma) .
$$

Although the case of $\gamma=0$ may look complicated, it follows immediately from Figure 5 (right) that the angle between the unit downward normal vector of $v^{+}$and $\vec{v}_{1}$ are parallel on the boundary, on $\partial \Omega_{1} \cap \partial \Omega_{0}$,

$$
\vec{v}_{1} \cdot T v^{+}=1=\cos 0 .
$$

Also it follows immediately from the definition of the differential operator $T$ that $\left|T v^{+}\right| \leq 1$; see (1-6). By noting that $\vec{v}_{2}$ is a unit vector, i.e., $\left|\vec{v}_{2}\right|=1$, we have

$$
\nu_{2} \cdot T v^{+}>-1=\cos (\pi-0) .
$$

Hence the surface $v^{+}(s, t)$ defined by (3-6)-(3-7) satisfies Inequalities (A-2) and (A-3). We now show that the surface $v^{+}(s, t)$ satisfies (A-1) by choosing large enough constant $K$.

Since $\nabla \cdot T v^{+}$is twice the mean curvature of the surface $v^{+}$, it is given by the well-known formula (see [Moon and Spencer 1970], for example)

$$
\nabla \cdot T v^{+}=-2 H\left(v^{+}\right)=-\frac{E N+G L-2 F M}{E G-F^{2}},
$$


where

$$
E=\left(x_{s}\right)^{2}+\left(y_{s}\right)^{2}+\left(z_{s}\right)^{2}, \quad F=x_{s} x_{t}+y_{s} y_{t}+z_{s} z_{t}, \quad G=\left(x_{t}\right)^{2}+\left(y_{t}\right)^{2}+\left(z_{t}\right)^{2},
$$

and

$$
L=\frac{\left|\begin{array}{ccc}
x_{s s} & y_{s s} & z_{s s} \\
x_{s} & y_{s} & z_{s} \\
x_{t} & y_{t} & z_{t}
\end{array}\right|}{\sqrt{E G-F^{2}}}, \quad M=\frac{\left|\begin{array}{ccc}
x_{s t} & y_{s t} & z_{s t} \\
x_{s} & y_{s} & z_{s} \\
x_{t} & y_{t} & z_{t}
\end{array}\right|}{\sqrt{E G-F^{2}}}, \quad N=\frac{\left|\begin{array}{ccc}
x_{t t} & y_{t t} & z_{t t} \\
x_{s} & y_{s} & z_{s} \\
x_{t} & y_{t} & z_{t}
\end{array}\right|}{\sqrt{E G-F^{2}}} .
$$

After some calculation we obtain

$$
\begin{aligned}
\nabla \cdot T v^{+}=\frac{g_{1}^{\prime \prime}(t)}{\left(1+\left(g^{\prime}(t)\right)^{2}\right)^{3 / 2}} & \\
& +\frac{f_{1}^{\prime \prime}(s)}{\left(1+\left(f_{1}^{\prime}(s)\right)^{2}\right)^{3 / 2}\left(1+t \frac{f_{1}^{\prime \prime}(s)}{\left(1+\left(f_{1}^{\prime}(s)\right)^{2}\right)^{3 / 2}}\right)} \frac{g^{\prime}(t)}{\sqrt{1+\left(g^{\prime}(t)\right)^{2}}} .
\end{aligned}
$$

Recalling that we have chosen the domain $\Omega_{0}$ so that (3-5) holds in $\Omega_{0}$ and that $f_{1}^{\prime \prime}(s) \in C^{2}\left(\left[0, \epsilon_{o}\right]\right)$, in order to show $\nabla \cdot T v^{+}$is bounded, all we need to show is that $g_{1}^{\prime \prime}(t) /\left(1+\left(g^{\prime}(t)\right)^{2}\right)^{3 / 2}$ is bounded, that is to say, the curvature of the curve $g(t)$ is bounded. For the case of $\gamma \neq 0$, we have chosen $g(t)$ to be a linear function, so $g^{\prime \prime}(t)$ is zero. For the case of $\gamma=0$, we have chosen $g(t)$ to be the part of a circle with radius $t_{0}$, so $g_{1}^{\prime \prime}(t) /\left(1+\left(g^{\prime}(t)\right)^{2}\right)^{3 / 2}=1 / t_{0}$. In either case, it follows that $\nabla \cdot T v^{+}$is bounded. We now consider the quantity $\nabla \cdot T v^{+}-v^{+}$, which can be written as

$$
\nabla \cdot T v^{+}-v^{+}=\nabla \cdot T v^{+}-(g(t)+K) .
$$

It follows immediately from the choice of $g(t)$ that it is bounded in the domain $\bar{\Omega}_{0}$ and also we have shown that twice the mean curvature $\nabla \cdot T v^{+}$is bounded and does not depend on $K$. Hence there exists a constant $K_{0}$ such that

$$
\nabla \cdot T v^{+}-v^{+}=\nabla \cdot T v^{+}-(g(t)+K) \leq 0 \quad \text { for all } K \geq K_{0} .
$$

Thus we have shown that the surface $v^{+}$satisfies the (A-1) when $K>K_{0}$.

We now put the last piece of the puzzle in place by showing $v^{+}$satisfies (A-4) for an appropriate choice of the constant $K$. Corollary A.1 implies that the capillary surface $u$ is bounded away from the cusp, hence it is bounded on

$$
\partial \Omega_{0} \backslash\left(\partial \Omega_{1} \cup \partial \Omega_{2} \cup\{(0,0)\}\right) .
$$

Since $g(t)$ is bounded in the domain $\bar{\Omega}_{0}$, there exists a constant $K_{1} \geq K_{0}$ such that $g(t)+K_{1}>u$ on $\partial \Omega_{0} \backslash\left(\partial \Omega_{1} \cup \partial \Omega_{2} \cup\{(0,0)\}\right)$. Thus the surface $v^{+}$satisfies (A-4) when $K=K_{1}$. 
We have shown that the surface $v^{+}(s, t)$ defined in (3-6)-(3-7) satisfies inequalities (A-1)-(A-4), so by the Concus-Finn comparison principle we have

$$
v^{+}(s, t) \geq u(x, y) \text { in } \Omega_{0} .
$$

Therefore the capillary surface at the cusp is bounded above when $\gamma_{1}+\gamma_{2}=\pi$ and each boundary $\left(\partial \Omega_{1}, \partial \Omega_{2}\right)$ has finite curvature near the cusp.

We can follow the similar steps for constructing the subsurface to show that this capillary surface is bounded below. We first construct a coordinate system such that one of the families of the coordinate curves is parallel curves of the boundary $\partial \Omega_{2}$ and another is perpendicular lines of the boundary $\partial \Omega_{2}$. Then choose a surface $v^{-}$so that the heigh only depends on the distance from $\partial \Omega_{2}$ which satisfies the contact angle condition exactly on $\partial \Omega_{2}$ and also it satisfies $\vec{v}_{1} \cdot T v^{-}-\cos \gamma \leq 0$. By choosing $v^{-}$to have the bounded height and the finite mean curvature, we can shift this surface downwards enough to satisfy $\nabla \cdot T v^{-}-v^{-} \geq 0$ in $\Omega_{0}$ and $v^{-} \leq u$ on $\partial \Omega_{0} \backslash\left(\partial \Omega_{1} \cup \partial \Omega_{2} \cup\{(0,0)\}\right)$. Then using the Concus-Finn comparison principle, we can prove that $u(x, y)$ is bounded below.

Thus by showing that there exist bounded sub- and supersolutions of the LaplaceYoung capillary surface equation, we have proven that the capillary surface is bounded if the contact angles of the boundaries are supplementary angles and boundaries have finite curvatures near the cusp.

\section{B. Proof of the continuity of the capillary surface when $\gamma_{1}+\gamma_{2}=\pi$.}

Theorem 3.2. If the capillary surface satisfies the conditions in Theorem 3.1, it is continuous at the cusp.

Proof. Having established the boundedness of the solution, we can use the methods of [Lancaster and Siegel 1996] to establish a parametric description of the surface, with parameter domain at first the unit disk. The above comparison surface is needed in proving Case 5 (page 173) in that reference. Assuming the surface is discontinuous at the corner implies that an arc of the unit circle corresponds to the points on the surface above the corner point. A change of coordinates allows us to use the half-unit disk as the parameter domain, where the boundary line segment corresponds to the points on the surface above the corner point. Following the proof of Step 3 (page 175) of [Lancaster and Siegel 1996], for two different heights, there are level curves going through the corner point, and this leads to a contradiction (last paragraph of page 175 of the same reference).

\section{Concluding remarks}

We have shown that the validity of the statement "[the capillary surface] rises with the same order like the order of contact of the two arcs, which form the cusp" 
[Scholz 2004] is not restricted to power-law cusps; it can be extended further. Our proof directly uses the the functions $f_{1}(x)$ and $f_{2}(x)$ without approximating them by series. This idea has given us an advantage in the sense that our leading order term expression gives clearer intuitive understanding of the relationship between the shape of the domain and the shape of the singular capillary surface. Also as shown in an Example in Subsection 2.4.1, our leading order term gives first three terms of the power series asymptotic expansion, owing to the fact we have avoided approximating the boundary by the power series.

Even though we have extended the results beyond power-series cusps, our results still suffer from certain restrictions, including (2-8)-(2-12). Also a complete asymptotic series solution maybe desirable in order to claim a complete understanding of the asymptotic behavior; however, this will require further assumptions to the boundary functions $f_{1}$ and $f_{2}$. The authors suspect that functions $f_{1}$ and $f_{2}$ of a form similar to the right-hand side of (2-27) can be potential candidates for a type of cusp for which a complete asymptotic series can be determined.

Also we have shown the previously unknown phenomenon of a bounded capillary surface in a cusp domain is possible when the contact angles of the two walls are supplementary (i.e., $\gamma_{1}+\gamma_{2}=\pi$ ). Although our proof covers most of the cases when the boundaries are smooth except at the cusp, the behavior of the capillary surface is unknown when the curvature of the boundary is not finite at the cusp. For example, it is unknown whether or not the capillary surface is bounded in a cusp domain bounded by $f_{1}=x^{3 / 2}$ and $f_{2}=-x^{3 / 2}$ when the contact angles of the two walls are supplementary.

The phenomenon that the capillary surface can be bounded or unbounded in a cusp domain depending on the contact angle can be interesting physically, as it indicates that a gradual change in the contact angle (e.g., by changing the temperature of the liquid) can cause a dramatic change in the liquid surface from unbounded to bounded. However, as the bounded capillary surface in a cusp domain only appears when the contact angles are exactly supplementary, it is not unknown to the authors how easily this phenomena can be observed through an experiment.

Thus we end this paper by remarking that the further exploration of singular capillary surfaces through theoretical, experimental and possibly numerical analyses is desired.

\section{Appendix: The Concus-Finn comparison principle}

In Sections 2C and 3A we have used the Concus-Finn comparison principle. We present it here for readers unfamiliar with it; see [Finn 1986, pages 110-113; 1989] for detailed discussions and proofs. We use the following formulation of the comparison principle: 
Theorem A.1 (supersolution). Let $u(x, y)$ be a solution of the boundary value problem (1-3)-(1-5) and let $\Omega_{0}$ be a subdomain of $\Omega$, with boundary $\partial \Omega_{0}$. Suppose a function $v^{+}(x, y)$ satisfies the inequalities

$$
\begin{aligned}
\nabla \cdot T v^{+}-v^{+} \leq 0 & \text { in } \Omega_{0}, \\
\vec{v}_{1} \cdot T v^{+}-\cos \gamma_{1} \geq 0 & \text { on } \partial \Omega_{1} \cap \partial \Omega_{0}, \\
\vec{v}_{2} \cdot T v^{+}-\cos \gamma_{2} \geq 0 & \text { on } \partial \Omega_{2} \cap \partial \Omega_{0}, \\
v^{+}(x, y) \geq u(x, y) & \text { on } \partial \Omega_{0} \backslash\left(\partial \Omega_{1} \cup \partial \Omega_{2} \cup\{(0,0)\}\right) .
\end{aligned}
$$

Then $v^{+}(x, y)$ is a supersolution of the boundary value problem (1-3)-(1-5), i.e.,

$$
v^{+}(x, y) \geq u(x, y) \quad \text { in } \Omega_{0} .
$$

A similar statement holds for subsolutions.

Also we make use of one of the corollaries of the comparison principle to construct an upper bound for the solution; see [Concus and Finn 1974] or pages 113114 of [Finn 1986].

Corollary A.1 (bound by hemispheres). Let $u(x, y)$ be a solution of the boundary value problem (1-3)-(1-5) and $B_{r_{0}}\left(x_{0}, y_{0}\right)$ a disk of radius $r_{0}>0$ centered at $\left(x_{0}, y_{0}\right)$. If $B_{r_{0}}\left(x_{0}, y_{0}\right) \subseteq \Omega$, then

$$
-\left(\frac{1}{r_{0}}+r_{0}\right) \leq u(x, y) \leq \frac{1}{r_{0}}+r_{0} \quad \text { in } B_{r_{0}}\left(x_{0}, y_{0}\right) .
$$

Recalling from (1-2) that the boundary is assumed to be of class $C^{3}$ away from the origin, it follows immediately from Corollary A.1 that $u(x, y)$ can only be unbounded at the origin (cusp).

\section{Acknowledgements}

The authors thank Professor Robert Finn of Stanford University for useful advice and interesting discussions during his visit to the University of Waterloo in May 2007.

Aoki thanks Professor John Wainwright of the University of Waterloo, whose comments during the author's master's degree thesis defence led to refinements in some of the results in Section 2. He also thanks the National Institute of Informatics, since part of this manuscript was prepared during his internship at the institute in 2010.

Both authors thank the University of Waterloo and the Natural Science and Engineering Council of Canada, which funded this research. 


\section{References}

[Aoki 2007] Y. Aoki, “Analysis of asymptotic solutions for cusp problems in capillarity”, Master's thesis, University of Waterloo, 2007, available at http://hdl.handle.net/10012/3352.

[Concus and Finn 1969] P. Concus and R. Finn, "On the behavior of a capillary surface in a wedge", Proc. Nat. Acad. Sci. USA 63:2 (1969), 292-299. Zbl 0219.76104

[Concus and Finn 1974] P. Concus and R. Finn, "On capillary free surfaces in a gravitational field", Acta Math. 132 (1974), 207-223. MR 58 \#32327c Zbl 0382.76005

[Finn 1986] R. Finn, Equilibrium capillary surfaces, Grundlehren der Math. Wiss. 284, Springer, New York, 1986. MR 88f:49001 Zbl 0583.35002

[Finn and Hwang 1989] R. Finn and J.-F. Hwang, "On the comparison principle for capillary surfaces”, J. Fac. Sci. Univ. Tokyo Sect. IA Math. 36:1 (1989), 131-134. MR 90h:35099 Zbl 0684. 35007

[King et al. 1999] J. R. King, J. R. Ockendon, and H. Ockendon, "The Laplace-Young equation near a corner”, Quart. J. Mech. Appl. Math. 52:1 (1999), 73-97. MR $2000 f: 76017$ Zbl 0932.76012

[Lancaster and Siegel 1996] K. E. Lancaster and D. Siegel, "Existence and behavior of the radial limits of a bounded capillary surface at a corner", Pacific J. Math. 176:1 (1996), 165-194. MR 98g:58030a Zbl 0866.76018

[Miersemann 1993] E. Miersemann, "Asymptotic expansion at a corner for the capillary problem: the singular case", Pacific J. Math. 157:1 (1993), 95-107. MR 93m:35039 Zbl 0796.76020

[Moon and Spencer 1970] P. Moon and D. E. Spencer, Field theory handbook: including coordinate systems, differential equations and their solutions, 2nd ed., Springer, Berlin, 1970. MR 89i:00026 Zbl 0097.39403

[Norbury et al. 2005] J. Norbury, G. C. Sander, and C. F. Scott, "Corner solutions of the LaplaceYoung equation", Quart. J. Mech. Appl. Math. 58:1 (2005), 55-71. MR 2006b:76017 Zbl 1064. 76020

[Scholz 2001] M. Scholz, Über das Verhalten von Kapillarfächen in Spitzen, Ph.D. thesis, Universität Leipzig, 2001, available at http://www.people.imise.uni-leipzig.de/markus.scholz/pdf/p1.pdf.

[Scholz 2004] M. Scholz, "On the asymptotic behaviour of capillary surfaces in cusps", Z. Angew. Math. Phys. 55:2 (2004), 216-234. MR 2005e:76023 Zbl 1058.35059

Received June 17, 2011. Revised December 16, 2011.

\section{YASUNORI AOKI}

Department OF ApPlied Mathematics

UNIVERSITY OF WATERLOO

200 University AVEnUe West

WATERLOO, ON N2L 3G1

CANADA

yaoki@uwaterloo.ca

\section{DAVID SIEGEL}

Department of Applied Mathematics

UNIVERSITY OF WATERLOO

200 UnIVERSITY AVENUE WEST

WATERLOO, ON N2L 3G1

CANADA

dsiegel@uwaterloo.ca 


\title{
PACIFIC JOURNAL OF MATHEMATICS
}

\author{
http://pacificmath.org \\ Founded in 1951 by \\ E. F. Beckenbach (1906-1982) and F. Wolf (1904-1989)
}

\section{EDITORS}

V. S. Varadarajan (Managing Editor)

Department of Mathematics

University of California

Los Angeles, CA 90095-1555

pacific@math.ucla.edu

Vyjayanthi Chari

Department of Mathematics

University of California

Riverside, CA 92521-0135

chari@math.ucr.edu

\section{Robert Finn}

Department of Mathematics Stanford University

Stanford, CA 94305-2125

finn@math.stanford.edu

Kefeng Liu

Department of Mathematics

University of California

Los Angeles, CA 90095-1555

liu@math.ucla.edu
Darren Long

Department of Mathematics

University of California

Santa Barbara, CA 93106-3080

long@math.ucsb.edu

Jiang-Hua Lu

Department of Mathematics

The University of Hong Kong

Pokfulam Rd., Hong Kong jhlu@maths.hku.hk

Alexander Merkurjev

Department of Mathematics

University of California

Los Angeles, CA 90095-1555

merkurev@math.ucla.edu
Sorin Popa

Department of Mathematics University of California

Los Angeles, CA 90095-1555 popa@math.ucla.edu

Jie Qing

Department of Mathematics

University of California

Santa Cruz, CA 95064

qing@cats.ucsc.edu

Jonathan Rogawski

Department of Mathematics

University of California

Los Angeles, CA 90095-1555

jonr@math.ucla.edu

\section{PRODUCTION}

pacific@math.berkeley.edu

\section{SUPPORTING INSTITUTIONS}

ACADEMIA SINICA, TAIPEI

CALIFORNIA INST. OF TECHNOLOGY INST. DE MATEMÁTICA PURA E APLICADA KEIO UNIVERSITY

MATH. SCIENCES RESEARCH INSTITUTE NEW MEXICO STATE UNIV.

OREGON STATE UNIV.

\author{
STANFORD UNIVERSITY \\ UNIV. OF BRITISH COLUMBIA \\ UNIV. OF CALIFORNIA, BERKELEY \\ UNIV. OF CALIFORNIA, DAVIS \\ UNIV. OF CALIFORNIA, LOS ANGELES \\ UNIV. OF CALIFORNIA, RIVERSIDE \\ UNIV. OF CALIFORNIA, SAN DIEGO \\ UNIV. OF CALIF., SANTA BARBARA
}

\author{
UNIV. OF CALIF., SANTA CRUZ \\ UNIV. OF MONTANA \\ UNIV. OF OREGON \\ UNIV. OF SOUTHERN CALIFORNIA \\ UNIV. OF UTAH \\ UNIV. OF WASHINGTON \\ WASHINGTON STATE UNIVERSITY
}

These supporting institutions contribute to the cost of publication of this Journal, but they are not owners or publishers and have no responsibility for its contents or policies.

See inside back cover or pacificmath.org for submission instructions.

The subscription price for 2012 is US \$420/year for the electronic version, and \$485/year for print and electronic.

Subscriptions, requests for back issues from the last three years and changes of subscribers address should be sent to Pacific Journal of Mathematics, P.O. Box 4163, Berkeley, CA 94704-0163, U.S.A. Prior back issues are obtainable from Periodicals Service Company, 11 Main Street, Germantown, NY 12526-5635. The Pacific Journal of Mathematics is indexed by Mathematical Reviews, Zentralblatt MATH, PASCAL CNRS Index, Referativnyi Zhurnal, Current Mathematical Publications and the Science Citation Index.

The Pacific Journal of Mathematics (ISSN 0030-8730) at the University of California, c/o Department of Mathematics, 969 Evans Hall, Berkeley, CA 94720-3840, is published monthly except July and August. Periodical rate postage paid at Berkeley, CA 94704, and additional mailing offices. POSTMASTER: send address changes to Pacific Journal of Mathematics, P.O. Box 4163, Berkeley, CA 94704-0163.

PJM peer review and production are managed by EditFLOW ${ }^{\mathrm{TM}}$ from Mathematical Sciences Publishers.

PUBLISHED BY PACIFIC JOURNAL OF MATHEMATICS

at the University of California, Berkeley 94720-3840

A NON-PROFIT CORPORATION

Typeset in LATEX

Copyright $(02012$ by Pacific Journal of Mathematics 


\section{PACIFIC JOURNAL OF MATHEMATICS}

Volume $257 \quad$ No. $1 \quad$ May 2012

Energy and volume of vector fields on spherical domains

FABiano G. B. BRito, ANDRÉ O. Gomes and Giovanni S. Nunes

Maps on 3-manifolds given by surgery

BOLDIZSÁR KALMÁR and ANDRÁS I. STIPSICZ

Strong solutions to the compressible liquid crystal system

Yu-Ming ChU, Xian-Gao LiU and XIAO LIU

Presentations for the higher-dimensional Thompson groups $n V$

Johanna HenNig and FranCESCo MatuCCI

Resonant solutions and turning points in an elliptic problem with oscillatory

boundary conditions

ALFONSO CASTRO and ROSA PARDO

Relative measure homology and continuous bounded cohomology of topological pairs

\section{Roberto Frigerio and CRISTINA PAgLiAnTINi}

Normal enveloping algebras

ALEXANDRE N. GrishKov, Marina RASSKazova and SALVATORE SICILIANO

Bounded and unbounded capillary surfaces in a cusp domain

YASUNORI AOKI and DAVID SIEGEL

On orthogonal polynomials with respect to certain discrete Sobolev inner product

Francisco Marcellán, Ramadan Zejnullahu, Bujar Fejzullahu and EDMUNDO HUERTAS

Green versus Lempert functions: A minimal example

PASCAL THOMAS

Differential Harnack inequalities for nonlinear heat equations with potentials under the Ricci flow

JIA-YONG WU

On overtwisted, right-veering open books

PAOLO LISCA

Weakly Krull domains and the composite numerical semigroup ring $D+E\left[\Gamma^{*}\right]$

JUNG WOOK LIM

Arithmeticity of complex hyperbolic triangle groups 